\title{
On the Statistical Methods of the Signal Significance Estimation in the Detection of the Signal from the Point Sources of Cosmic Rays
}

\author{
A. Chilingarian, G.Hovsepyan, G.Gharagyozyan and G.Karapetyan \\ Alikhanyan Physics Institute, Yerevan, Armenia, Alikhanyan Brothers 2, Yerevan 375036, Armenia \\ Presenter: G.Hovsepyan (hgg@crdlx5.yerphi.am), arm-hovsepyan-G-abs3-og12-poster
}

The estimation of the significance of the peaks in 1 and 2-dimensional distributions is one of the most important problems in high-energy physics and astrophysics. The physical inference from low-statistics and, therefore, low-significance experiments usually is biased and many discoveries lack further confirmation. One of the typical mistakes in physical inference is the use of non-adequate statistical models. We demonstrate that extremum statistical models are more appropriate for estimating the significance of anisotropy studies, new particles finding, etc. We analyze the significance of the experimental evidence in the on-going efforts of detecting the point source of Cosmic Rays. The analysis is carried out in the framework of two models utilizing the extremum statistics: first - using the fixed grid of celestial coordinates, and second - using the tuned grid. The test distributions for the significance estimation are obtained both from simulation models and from the analytical treatment of the problem. We show that the second model gives adequate physical inference, while the first model can lead to the positively biased estimates of the point source significance.

\section{Introduction}

All sky searches of the Cosmic Ray sources is one of the most promising ways to gain insight in the longstanding problem of the origin of these particles. While many experiments have shown that the distribution of arrival directions are isotropic (see for example [1] and references therein), existence of the small-angle anisotropies has been climbed by several groups in "knee" energy region $10^{14}-10^{16} \mathrm{eV}[2,3]$ and for the ultrahigh energy range $>10^{19} \mathrm{eV}$.[4].

Physicists, observing positive fluctuation considerably greater than expected statistically usually ascribe this effect to a "source". However, as mentioned by Michael Hillas [5], our experience has shown that large excesses up to $6 \sigma$ are more common than were expected. When consistent and reliable statistical test are applied we can't obtain convincing prove for point sources. In 1973 there was demonstrated [6], that the evidence of many of claimed $\gamma$-ray sources when properly treated is rather weak.

In present publication we use both analytical approach and Monte-Carlo method to obtain the statistical model adequately describing signal searches with MAKET-ANI array[7]. The sources of possible erroneous physical inference based on biased models are discussed.

\section{Gaussian approximation}

In the experiment we measure the horizontal coordinates of the incident particle - zenith angle $\varphi$ and azimuth $\theta$, and then transform them to equatorial coordinates - right ascension RA and declination $\alpha$, according to transformation equations [8]. In our probabilistic treatment of the problem we convolute the uniform distribution of RA and treat number of events hitting different bins as realizations of the multinomial random process with $\bar{N}_{j}, \mathrm{j}=1,20$ fixed probabilities. Then, by normalization of the bin contain 
according to the mean and variance of corresponding declination belt we obtain standard Gaussian distribution $\mathrm{N}(0,1)$ to be used further as the test statistics:

$$
\sigma_{i, j}=\frac{N_{i, j}-\bar{N}_{j}}{\sqrt{\bar{N}_{j}}}, \quad i=1, N_{\alpha}, 3, \quad j=N_{\delta 1, \delta 2,3}
$$

where $\mathrm{N}_{\mathrm{i}, \mathrm{j}}$ is the number of events in the rectangular bins, $\bar{N}_{j}$ and $\sqrt{\bar{N}_{j}}$ - RA averaged mean and mean square deviation of number of events fallen in the bin of $\mathrm{j}^{\text {th }}$ belt, $\mathrm{N}_{\alpha}=360$ is maximal value of $\mathrm{RA} ; \mathrm{N}_{\delta 1}=6.6$ is the first declination, $\mathrm{N}_{\delta 2}=66.6$ is the last declination for a total 20 declination belts, each of $3^{\circ}$. We were looking for Single Source candidates in the two-dimensional $\Delta \alpha \times \Delta \delta\left(3^{\circ} \times 3^{\circ}\right)$ grid, covering a $360^{\circ} \times 60^{\circ}$ equatorial coordinate range with $\mathrm{M}=2400$ bins. The origin of the rectangular equatorial coordinate system (grid) was taken at $\left(0^{\circ}, 6.6^{\circ}\right)$. Of course, the multinomial significances are different from the Gaussian ones [9] especially for the large significance values, nevertheless first we will present results with the commonly used Gaussian distribution. The introduced bias due to slow convergence of Gaussian approximation toward the correct multinomial (Poissonian) values will be discussed elsewhere.

More than 2 million particle showers detected by the MAKET-ANI detector, with sizes starting from $\mathrm{N}_{\mathrm{e}}$ $>10^{5}$ electrons were distributed among the $\mathrm{M}=2400$ angular bins. The signal was revealed when we examined the sub-sample of $\sim 6 \cdot 10^{4}$ events with $\mathrm{N}_{\mathrm{e}}>10^{6}$.

According to the logic of hypothesis testing we calculate the test statistics by applying equation (1) to the experimentally detected showers and using a fixed equatorial grid. From the obtained value of 6.04 for the "signal bin" we have calculated corresponding probability of obtaining this value under $\mathrm{H}_{0}$ hypothesis.

We assume that maximal obtained value for the signal bin 6.04 belongs to $\mathrm{N}(0,1)$ distribution. Therefore, the probability density distribution function of obtaining this value as the maximal value among $\mathrm{M}$ possibilities is straightaway [10]:

$$
P_{M}(x)=M \cdot g(x)\left(1-G_{>x}\right)^{M-1} \approx M \cdot g(x) \cdot e^{-M \cdot G_{>x}}
$$

Where $\mathrm{g}(\mathrm{x})$ is standard Gaussian probability density for the signal bin;

$\mathrm{G}_{>\mathrm{x}}=\int_{x}^{\infty} g(t) d t$ is so called test statistics p-value: the probability to obtain the value of test statistics in the interval greater than $\mathrm{x}$.

To obtain the chance probability to observe in one from 2400 bins number of events equivalent or more than 6.04 standard deviations ( $p$-value of the distribution $\mathrm{P}_{\mathrm{M}}(\mathrm{x})$ ) we have to integrate $\mathrm{P}_{M}(x)$ in the interval [6.04, $+\infty)$. For $\mathrm{M}=2400$ we obtain $\int_{6.04}^{\infty} P_{M}(x) d x \sim 2 \cdot 10^{-6}$. Proceeding from this very small value, we rejected the null hypothesis and concluded that the MAKET-ANI has detected signal from the direction of the Monogem Ring.

\section{Bin regrouping effect}

The physicists usually slightly adjust the grid superimposed on the sky map, to include as much as possible of the "signal "events in selected bin as possible. Each regrouping in order to maximize the effect leads to increase of $\mathrm{M}$ in formula (2) thus changing the chance probability. To check and enumerate these changes 
we develop two simulation models. Our first model generates the random Gaussian variables in 120 RA bins of each of 20 declination belts according to the belt-specific means and variances as obtained in the experiment. After applying normalizing transformation (1) to the generated random map we obtained $\mathrm{M}=2400$ random variables distributed according standard Gaussian $\mathrm{N}(0,1)$. Then the maximal positive deviation from the $\mathrm{N}(0,1)$ was stored as value of the test statistics. After more than 1000 independent random experiments with the model we obtained the distribution of the extremum statistics.

Our second model generates a number of events in the same way as the first one. Then the origin of equatorial coordinate system (right ascension and declination) is shifted by $0.1^{\circ}$ in each dimension in the range equal to one bin size $\left(3^{\circ} \times 3^{\circ}\right)$. Those instead of one grid 900 different grids are tested. The goal of this procedure is to obtain maximal possible "signal" for given distribution of events. After shift, the current value of extremum is compared with the previous best one, and if the new one is larger, it is saved.
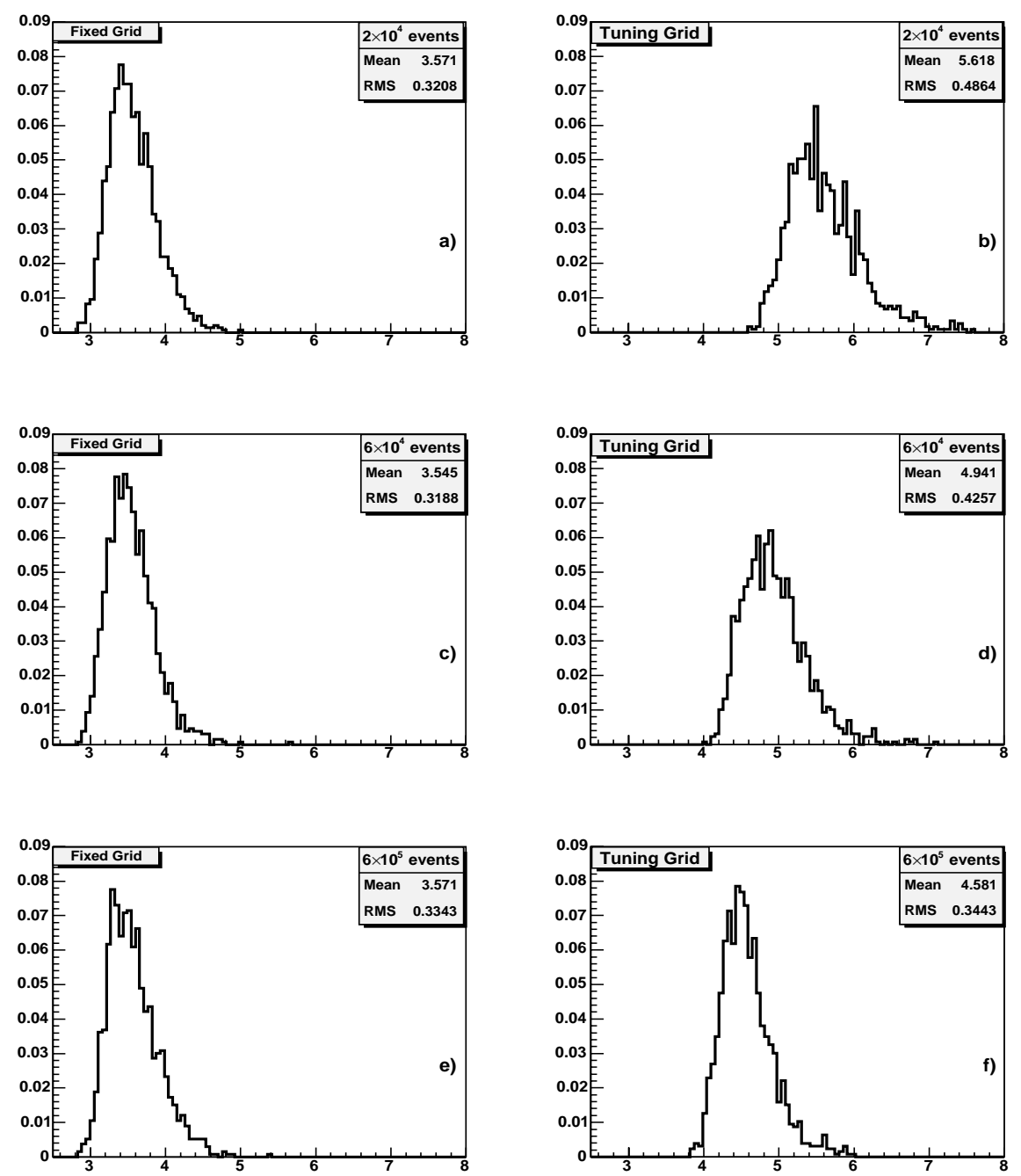

Figure 1. Distribution of the extremum test statistics values for the first simulation model - one fixed grid with $\mathrm{M}=2400$ bins - left column, and for second simulation model - with tuning the grid to contain maximal signal - right column. 
The maximal values selected by single fixed grids fit very well to the theoretical expectations (2) with value of $\mathrm{M}=2400$. The actual chance probability (test $p$-value correspondent to the $\sigma \sim 6$ ) obtained with first model [3], as we can see from the Figure 1a), c), e) is vanishingly small and evidence of signal - very strong.. The second way of data analysis, in contrast to the first one, produces the extremum distributions significantly shifted to the large values of $\sigma$. Therefore, the test p-value is relatively large, especially for small number of events; see Figure 1, b), d), f) and the evidence of signal became rather weak. Note the excellent agreement of test statistics distribution obtained from f-la (2) with $\mathrm{M}=2400$ and distribution obtained with Monte-Carlo method.

When we are testing numerous grids the distribution mean is significantly shifted to the higher values, and probability to obtain large extremum values is dramatically enlarged, especially for the low statistics experiments. For the MAKET - ANI statistics of $\sim 6 \cdot 10^{4}$ events with $\mathrm{N}_{\mathrm{e}}>10^{6}$, the shift of the mean is $\sim 1.5$ and we can easily obtain significance values exceeding 6 and even 7 .

\section{Conclusions}

1. In estimating the significance of the signal detection we are looking for the maximal value of deviation from the normalized belt means, and statistical inference is drawn based on the value of this maximum. Therefore, the extreme statistics distribution (2) should be used as the test statistics for estimating the significance of signal;

2. Both analytical distribution (2) and simulated distribution obtained with Monte Carlo method give very consistent results, proving necessity to account on all choices of data grouping aimed to reveal signal.

\section{Acknowledgements}

We thank Suren Chilingarian for writing code and performing simulations with the first and second models of point source detection, Artur Reimers for calculating significance levels and making Figures 10 and 11.

\section{References}

[1] T. Antony et al., (KASCADE Collaboration), ApJ, 608, 865(2003).

[2] M. Samorski, W.Stamm, ApJ, 268, L17 (1983).

[3] A. Chilingarian, H.Martirosian, G. Gharaghoyzyan, ApJl, 597, pp. L129-131(2003).

[4] M. Takeda et al., ApJ 522, 225(1999).

[5] M. Hillas, Proc. $14^{\text {th }}$ ICRC, 9, 3439(1975).

[6] E. O’Mongain, Nature 241, 376(1973).

[7] V. Avakian, E.V. Bazarov et. al., VANT, Series in Technical Physics Experiments, 5(31), p.1(1986).

[8] J.Meeus, Astronomical Algorithms, Willmann-Bell Inc. (1991).

[9] H.Ebeling, astro-ph/0301285(2003).

[10] S.C. Chapman, G.Rowlands, N.W.Watkins, Nonlinear Processes in Geophysics, 9, 409-418(2002) 